\title{
Avaliação da Percepção do Paladar de Pacientes Oncológicos: Relação com Variáveis Pessoais e Clínicas e Comparação com um Grupo Controle
}

doi: https://doi.org/10.32635/2176-9745.RBC.2021v67n1.994

\author{
Evaluation of Taste Perception of Oncological Patients: Relationship with Personal and Clinical Variables and Comparison \\ with a Control Group \\ Evaluación de la Percepción del Sabor de Pacientes Oncológicos: Relación con Variables Personales y Clínicas y Comparación \\ con un Grupo de Control
}

\author{
Fabíola Pansani Maniglia'; Leonice Caldeira da Cruz²; Lauane Cristina Marques Costa ${ }^{3}$; Lara Coelho de Oliveira Silva ${ }^{4}$; Bruno Affonso \\ Parenti de Oliveira ${ }^{5}$
}

\section{RESUMO}

Introduçáo: $\mathrm{O}$ tratamento antineoplásico pode causar efeitos colaterais que prejudicam a ingestão alimentar e o estado nutricional dos pacientes. Objetivo: Avaliar a percepção do paladar de pacientes em tratamento oncológico, relacioná-la com as variáveis pessoais e clínicas e compará-la com a percepção do paladar de indivíduos sadios. Método: Trata-se de um estudo transversal com 50 indivíduos em tratamento oncológico e outros 50 indivíduos do grupo controle. Para avaliar a percepçáo do paladar, os participantes receberam amostras de alimentos e foram questionados quanto aos sabores doce, amargo, salgado e azedo. A intensidade do sabor foi avaliada por meio de uma escala de 1 a 5. Resultados: Os pacientes em tratamento oncológico perceberam os sabores dos seguintes alimentos de maneira

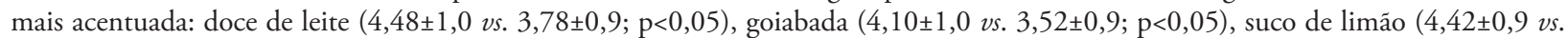
$2,86 \pm 1,0 ; \mathrm{p}<0,05)$, suco de maracujá $(3,76 \pm 1,2$ vs. $2,56 \pm 1,0 ; \mathrm{p}<0,05)$ e café $(4,42 \pm 0,8$ vs. $3,14 \pm 0,9 ; \mathrm{p}<0,05)$. Já os alimentos: biscoito de polvilho, iogurte natural, rúcula e chicória foram percebidos com menor intensidade quando comparados ao grupo controle. Entre as quatro categorias de sabor avaliadas, os alimentos do grupo salgado foram os que menos se diferenciaram quanto à percepção do paladar e os alimentos azedos e amargos foram percebidos de forma mais distinta entre os grupos. Conclusáo: O presente estudo é pioneiro em avaliar a percepção do paladar utilizando alimentos, ao invés de soluções padronizadas; e, por meio dessa técnica, identificaram-se diferenças significativas na intensidade da percepção do paladar dos indivíduos em tratamento oncológico.

Palavras-chave: Neoplasias; Oncologia; Antineoplásicos/efeitos adversos; Paladar; Disgeusia.

\section{ABSTRACT}

Introduction: Antineoplastic treatment can cause side effects that impair the patients' food intake and nutritional status. Objective: To evaluate the taste perception in patients undergoing cancer treatment, to relate it to personal and clinical variables and to compare with taste perception in healthy individuals. Method: Cross-sectional study with 50 individuals undergoing cancer treatment and another 50 individuals in the control group. To assess taste perception, participants received food samples and were asked about sweet, bitter, salty and sour flavors. The intensity of the flavor was assessed using a scale from 1 to 5. Results: Patients undergoing cancer treatment perceived the flavors of the following foods more strongly: dulce de leche $(4.48 \pm 1.0$ vs. $3.78 \pm 0.9 ; \mathrm{p}<0.05)$, guava $(4.10 \pm 1.0$ vs. $3.52 \pm 0.9 ; \mathrm{p}<0.05)$, lemon juice (4.42 \pm 0.9 vs. $2.86 \pm 1.0 ; \mathrm{p}<0.05)$, passion fruit juice $(3.76 \pm 1.2$ vs. $2.56 \pm 1.0$; $\mathrm{p}<0.05)$ and coffee $(4.42 \pm 0.8 v$ s. $3.14 \pm 0.9 ; \mathrm{p}<0.05)$. The foods: cassava starch, natural yogurt, arugula and chicory were perceived with less intensity when compared to the control group. Among the four flavor categories evaluated, the foods in the salty group were the ones that least differed in terms of the taste perception and sour and bitter foods were perceived more distinctly between the groups. Conclusion: The present study is pioneer in evaluating the taste perception using food, instead of standardized solutions, and through this technique, significant differences were identified in the intensity of taste perception of individuals undergoing cancer treatment.

Key words: Neoplasms; Medical Oncology; Antineoplastic Agents/adverse effects; Taste; Dysgeusia.

\section{RESUMEN}

Introducción: El tratamiento antineoplásico puede causar efectos secundarios que perjudican la ingesta de alimentos y el estado nutricional de los pacientes. Objetivo: Evaluar la percepción del gusto en pacientes sometidos a tratamiento contra el cáncer, relacionarlo con variables personales y clínicas y compararlo con la percepción del gusto en individuos sanos. Método: Este es un estudio transversal con 50 individuos en tratamiento contra el cáncer y otros 50 individuos en el grupo de control. Para evaluar la percepción del sabor, los participantes recibieron las muestras de alimentos y se les preguntó sobre los sabores dulces, amargos, salados y agrios. La intensidad del sabor se evaluó usando una escala del 1 al 5. Resultados: Los pacientes en tratamiento contra el cáncer percibieron los sabores de los siguientes alimentos con más fuerza: dulce de leche $(4,48 \pm 1,0$ vs. $3,78 \pm 0,9 ; \mathrm{p}<0,05)$, guayaba $(4,10 \pm 1,0$ vs. $3,52 \pm 0,9$; $\mathrm{p}<0,05)$, jugo de limón $(4,42 \pm 0,9$ vs. $2,86 \pm 1,0 ; \mathrm{p}<0,05)$, jugo de maracuyá $(3,76 \pm 1,2$ vs. $2,56 \pm 1,0 ; \mathrm{p}<0,05)$ y café $(4,42 \pm 0,8$ vs. $3,14 \pm 0,9 ; \mathrm{p}<0,05)$. Los alimentos: galletas de almidón, yogur, rúcula y achicoria se percibieron con menos intensidad en comparación con el grupo de control. Entre los sabores, los alimentos salados fueron los que menos diferían y los alimentos amargos y amargos se percibieron más claramente entre los grupos. Conclusión: El presente estudio es pionero en evaluar la percepción del gusto utilizando alimentos, en lugar de soluciones estandarizadas, y identificó diferencias significativas en la intensidad de la percepción del gusto en individuos en tratamiento contra el cáncer.

Palabras clave: Neoplasias; Oncología Médica; Antineoplásicos/efectos adversos; Gusto; Disgeusia.

\footnotetext{
1,2,3 Universidade de Franca (Unifran). Franca (SP), Brasil.

${ }^{4}$ Hospital do Câncer de Franca. Franca (SP), Brasil.

5Universidade de São Paulo (USP). Faculdade de Medicina de Ribeirão Preto (FMRP). Ribeirão Preto (SP), Brasil.

'E-mail: fa_nutricao@hotmail.com. Orcid iD: https://orcid.org/0000-0002-3281-9470

2E-mail: leonicecruzcaldeira@outlook.com. Orcid iD: https://orcid.org/0000-0001-8754-3937

${ }^{3}$ E-mail: lauane.mcosta@hotmail.com. Orcid iD: https://orcid.org/0000-0001-6791-2942

4E-mail: lara.30cos@gmail.com. Orcid iD: https://orcid.org/0000-0001-9929-8158

${ }^{5}$ E-mail: bruno_parenti@hotmail.com. Orcid iD: http://orcid.org/0000-0002-4233-5517

Endereço para correspondência: Fabíola Pansani Maniglia. Avenida Dr. Armando de Sales Oliveira, 201 - Parque Universitário. Franca (SP), Brasil. CEP 14404-600. E-mail: fa_nutricao@hotmail.com
} 


\section{INTRODUÇÃO}

A cada ano, cerca de 12,7 bilhôes de pessoas no mundo são diagnosticadas com câncer. Estimativas apontam que até 2030 ocorrerá 1,7 milhão de novos casos da doença na Região da América Latina e do Caribe ${ }^{1}$. No Brasil, a projeção, para cada ano do triênio 2020-2022, é de 625 mil novos casos de neoplasias malignas ${ }^{2}$.

O câncer é caracterizado pelo crescimento desordenado das células e, por isso, é também denominado de neoplasia maligna. Tal crescimento celular pode fugir parcial ou totalmente ao controle do organismo, invadindo tecidos e órgãos e causando efeitos agressivos ao paciente ${ }^{3}$. Trata-se de uma doença crônica não transmissível (DCNT), cuja evoluçáo epidemiológica está associada a fatores como processo de urbanização, industrialização, consumo de bebidas alcoólicas, inatividade física, alimentação inadequada, tabagismo e demais fatores ambientais ${ }^{4}$.

$\mathrm{O}$ tratamento para as neoplasias malignas são cirurgia, radioterapia e quimioterapia, podendo ou não serem associadas. Enquanto a cirurgia consiste na retirada da massa tumoral, a radioterapia é um método capaz de eliminar as células tumorais por meio da radiação ionizante aplicada diretamente no local do tumor. Já a quimioterapia é uma modalidade de tratamento que envolve o uso de substâncias citotóxicas administradas de maneira endovenosa e pode ser classificada de acordo com a sua finalidade ${ }^{5}$. Além do objetivo de atingir as células malignas, a quimioterapia também agride as células normais e causa diversos efeitos colaterais que comprometem a qualidade de vida do paciente, como náuseas, vômitos, alopecia, lesão esofágica, desequilíbrio hidroeletrolítico e alteração do paladar. Este último, conhecido como disgeusia, compromete ainda mais a ingestáo alimentar dos pacientes e colabora para a deterioração do estado nutricional ${ }^{6,7}$.

Sabe-se que o câncer acomete mais pessoas em idade avançada, período da vida em que o estado nutricional já pode se encontrar fragilizado, inclusive por alteraçóes na própria percepçáo do paladar ${ }^{8,9}$. O declínio do número de papilas gustativas e até de enzimas digestivas acabam por aumentar a disgeusia e comprometer a ingestão alimentar? No entanto, não são só os idosos doentes que sofrem com a disgeusia.

Pesquisadores sugerem diferentes mecanismos que podem influenciar na percepçáo do paladar pelo paciente em tratamento oncológico, iniciando-se pela inflamaçáo que o acomete, caracterizada pelas citocinas que modificam não só as sensaçóes gustativas, mas também olfativas. A deficiência do micronutriente zinco também é apontada como facilitadora da diminuição do paladar, assim como a xerostomia, que consiste na diminuição da salivação, também provocada pelo tratamento oncológico.
Vale ressaltar que as medidas antineoplásicas por si só levam à apoptose celular e contribuem para essa situaçãó ${ }^{10}$.

Reconhecendo que todas essas causas da disgeusia influenciarão na ingestão alimentar, pesquisadores vêm estudando o paladar de pacientes oncológicos. Pugnaloni et al. ${ }^{11}$ investigaram as alteraçóes na percepção dos sabores salgado, doce, azedo e amargo, utilizando soluçôes de diferentes concentrações. Os autores constataram que houve diferença significativa na percepção do paladar entre o grupo de pacientes oncológicos e o grupo controle ${ }^{11}$.

Até o momento, desconhecem-se estudos que tenham realizado tal investigação usando os próprios alimentos, ao invés de soluçóes com os sabores básicos. Por esse motivo, o presente estudo tem como objetivo avaliar a percepção do paladar de pacientes em tratamento oncológico, associá-la com as variáveis clínicas e antropométricas e compará-la com a percepção do paladar de indivíduos sadios.

\section{MÉTODO}

Esta pesquisa foi realizada com pacientes em tratamento oncológico no Hospital do Câncer do Complexo Santa Casa de Misericórdia de Franca, no período de agosto a dezembro de 2019, após a aprovação do Comitê de Ética em Pesquisa dessa mesma instituição, sob o número CAAE 17466319.8.0000.5438, e possui registro brasileiro de ensaio clínico RBR-94857q.

Trata-se de um estudo de intervenção, no qual foram recrutados 50 indivíduos que preencheram os seguintes critérios de inclusão: idade igual ou superior a 18 anos, estar em tratamento oncológico, com capacidade de se alimentar via oral, e que manifestaram o interesse em participar da pesquisa assinando o Termo de Consentimento Livre e Esclarecido (TCLE). Foram adotados os seguintes critérios de exclusão: pacientes tabagistas ou com localização da neoplasia em região de cabeça e pescoço.

Para compor o grupo controle, foram recrutados outros 50 indivíduos por meio de convite verbal aos funcionários da universidade promotora do estudo. Consideraram-se como critérios de inclusão: indivíduos sadios, não tabagistas e que também assinaram o TCLE. Para esse grupo, o critério de exclusáo se restringiu ao tabagismo. Esses indivíduos foram recrutados em um momento posterior à intervenção com os pacientes oncológicos, com o intuito de parear os grupos quanto às características de sexo e idade.

O número determinado de sujeitos visava a alcançar resultados mais significativos, uma vez que não seria possível eleger pacientes com um só tipo de neoplasia ou em uso de um único medicamento, no intuito de homogeneizar a amostra. 
No primeiro encontro com a pesquisadora, os pacientes oncológicos receberam um questionário alimentar, o qual auxiliou no conhecimento sobre os hábitos alimentares pregressos e atuais, as preferências de consistência alimentar e as aversóes e intolerâncias. Os dados clínicos como diagnóstico médico, tipo de neoplasia, medicamentos e tempo de tratamento foram obtidos em prontuário médico. Além dos dados clínicos, foi realizada a avaliaçáo antropométrica composta da obtenção de peso, estatura e circunferências da panturrilha $(\mathrm{CP})$, cintura e braço $(\mathrm{CB})$.

Feita a descrição da população do estudo, foi iniciada a intervenção na sala de quimioterapia do Serviço. Inicialmente, foram solicitados que os pacientes fizessem bochecho com água por duas vezes para limpar a cavidade oral. Em seguida, eles receberam as amostras de alimentos e foram questionados quanto à percepçáo dos sabores doce, amargo, salgado e azedo (nesta ordem). Para isso, foram usados três alimentos de cada um dos quatro sabores na quantidade de 5 gramas ou $5 \mathrm{~mL}$. Os alimentos doces foram: doce de leite (1), goiabada (2) e mel (3); os amargos: rúcula (1), chicória (2) e café (3); os salgados: biscoito de sal (1), biscoito de polvilho (2) e azeitona sem caroço (3); e os azedos: suco de limão (1), suco de maracujá (2) e iogurte natural integral (3). Os alimentos foram escolhidos pelas pesquisadoras, considerando que são presentes na alimentação da população brasileira e de fácil acesso.

A intensidade do sabor foi avaliada por meio de uma escala não validada de 1 a 5 , sendo 1 referente à mínima intensidade e 5 à máxima intensidade. Entre a avaliação de cada amostra, foi feito bochecho com água com o propósito de não influenciar na percepção do gosto do próximo alimento. A mesma intervenção foi realizada com o grupo controle.

A estatística descritiva foi realizada por meio dos valores de média e desvio-padrão. O teste de Komogorov-Smirnov foi utilizado para verificar a normalidade dos dados. Para verificar diferença entre os dois grupos do estudo, foi utilizado o teste t de Student. A análise de variância (ANOVA) com post hoc de Bonferroni foi utilizada para identificar as diferenças na escala de intensidade entre os três alimentos do mesmo grupo. Todas as análises foram realizadas com o software SPSS, versão 20, e nível de significância adotado de $\mathrm{p}<0,05$.

\section{RESULTADOS}

Participaram do estudo 50 pacientes em tratamento oncológico e 50 indivíduos sadios que compuseram o grupo controle. A média da idade dos integrantes da pesquisa foi de $57,8 \pm 12,0$ anos para o grupo de pacientes oncológicos e 58,1 $\pm 12,6$ para o grupo controle.
Entre os indivíduos em tratamento oncológico, o tipo de câncer predominante nas mulheres foi o de mama; enquanto, nos homens, foi localizado no intestino grosso. Todos eles faziam quimioterapia e dez deles também eram submetidos à radioterapia em locais que não incluíam as regiôes de cabeça e pescoço. Vale mencionar que não houve associação entre a localização do tumor e os medicamentos antineoplásicos com a percepção do paladar.

As demais características pessoais e nutricionais dos participantes estão apresentadas na Tabela 1 .

Houve diferença estatística entre os indivíduos em tratamento oncológico e o grupo controle, respectivamente, nas variáveis: índice de massa corporal - IMC $(25,9 \pm 4,5$ vs. $28,1 \pm 5,6 ; \mathrm{p}<0,05), \mathrm{CP}(34,0 \pm 3,5$ vs. $37,8 \pm 4,2 ; \mathrm{p}<0,01)$ e $\mathrm{CB}(28,0 \pm 3,6$ vs. $31,8 \pm 4,8 ; \mathrm{p}<0,01)$. No entanto, não houve associaçáo entre as variáveis do estado nutricional e a percepçáo dos sabores pelos pacientes em tratamento oncológico.

A Tabela 2 mostra a intensidade dos sabores percebida em cada alimento pelos pacientes e integrantes do grupo controle.

Entre as quatro categorias de sabor avaliadas, os alimentos do grupo salgado foram os que menos se diferenciaram quanto à percepção do paladar, enquanto os alimentos azedos e amargos foram percebidos de forma mais distinta entre os grupos controle e de pacientes em tratamento oncológico.

Destaca-se que, entre os 12 alimentos degustados, dez tiveram o seu sabor avaliado na mais alta intensidade pelos pacientes em tratamento oncológico.

Quando avaliadas as diferenças entre os alimentos de uma mesma categoria, observou-se que o grupo controle percebeu o sabor doce do mel significativamente superior ao sabor doce dos alimentos doce de leite e goiabada. Isto não ocorreu com o grupo de pacientes em tratamento; ou seja, os pacientes oncológicos náo sentiram diferença na intensidade do sabor doce entre os alimentos mel, doce de leite e goiabada.

Os alimentos biscoito salgado e azeitona e biscoito de polvilho e azeitona tiveram a intensidade do sabor salgado percebida de maneira distinta em ambos os grupos, sendo que a azeitona foi percebida como a mais salgada.

O grupo de alimentos azedos foi o único que apresentou diferença estatisticamente significativa na percepção do sabor entre os dois grupos e em todas as comparaçóes, sendo o iogurte considerado o alimento mais azedo pelo grupo controle e o menos azedo pelos pacientes em tratamento. Curiosamente, entre os alimentos do grupo amargo, o café foi classificado como o alimento menos amargo pelo grupo controle e o mais amargo pelos pacientes oncológicos. 
Tabela 1. Características demográficas e nutricionais da população. Franca (SP), 2019

\begin{tabular}{|c|c|c|c|c|c|c|}
\hline \multirow[t]{2}{*}{ Variável } & \multicolumn{2}{|c|}{ Pacientes $(n=50)$} & \multicolumn{2}{|c|}{ Controle $(n=50)$} & \multicolumn{2}{|c|}{ Total $(n=100)$} \\
\hline & $\mathbf{N}$ & $\%$ & $\mathbf{N}$ & $\%$ & $\mathbf{N}$ & $\%$ \\
\hline \multicolumn{7}{|l|}{ Sexo } \\
\hline Feminino & 35 & 70 & 35 & 70 & 70 & 70 \\
\hline Masculino & 15 & 30 & 15 & 30 & 30 & 30 \\
\hline \multicolumn{7}{|l|}{ Idade } \\
\hline 29 a 40 anos & 6 & 12 & 6 & 12 & 12 & 12 \\
\hline 41 a 59 anos & 21 & 42 & 21 & 46 & 42 & 42 \\
\hline 60 a 70 anos & 14 & 28 & 13 & 22 & 27 & 27 \\
\hline 71 a 85 anos & 9 & 18 & 10 & 20 & 19 & 19 \\
\hline \multicolumn{7}{|l|}{ IMC adulto } \\
\hline Baixo peso & 2 & 4 & 0 & 0 & 2 & 2 \\
\hline Eutrofia & 9 & 18 & 7 & 14 & 16 & 16 \\
\hline Excesso de peso & 16 & 32 & 20 & 40 & 36 & 36 \\
\hline \multicolumn{7}{|l|}{ IMC idoso } \\
\hline Baixo do peso & 5 & 10 & 2 & 4 & 7 & 7 \\
\hline Eutrofia & 10 & 20 & 8 & 16 & 18 & 18 \\
\hline Excesso de peso & 8 & 16 & 13 & 26 & 21 & 21 \\
\hline \multicolumn{7}{|l|}{ СB } \\
\hline Sem risco & 19 & 38 & 27 & 54 & 46 & 46 \\
\hline Com risco & 29 & 58 & 20 & 40 & 49 & 49 \\
\hline Sem classificação & 2 & 4 & 3 & 6 & 5 & 5 \\
\hline \multicolumn{7}{|l|}{$\mathrm{CP}$} \\
\hline Adequada & 38 & 76 & 47 & 94 & 85 & 85 \\
\hline Inadequada & 12 & 24 & 3 & 6 & 15 & 15 \\
\hline
\end{tabular}

Legendas: IMC: Índice de massa corporal; CB: Circunferência do braço; CP: Circunferência da panturrilha.

Tabela 2. Valores de média e desvio-padrão da intensidade da percepção dos sabores doce, amargo, salgado e azedo pelos participantes do estudo $(n=50)$. Franca (ŚP), 2019

\begin{tabular}{|c|c|c|}
\hline Grupo de alimentos & $\begin{array}{c}\text { Pacientes } \\
(n=50)\end{array}$ & $\begin{array}{c}\text { Controles } \\
(n=50)\end{array}$ \\
\hline \multicolumn{3}{|l|}{ Doce } \\
\hline 1 Doce de leite & $4,48 \pm 1,0$ & $3,78 \pm 0,9 *$ \\
\hline 2 Goiabada & $4,10 \pm 1,0$ & $3,52 \pm 0,9 * a$ \\
\hline $3 \mathrm{Mel}$ & $4,40 \pm 0,9$ & $4,32 \pm 0,8^{b c}$ \\
\hline \multicolumn{3}{|l|}{ Amargo } \\
\hline 1 Rúcula & $3,64 \pm 1,3$ & $4,46 \pm 0,9^{*}$ \\
\hline 2 Chicória & $3,00 \pm 1,1^{a}$ & $3,86 \pm 1,0 * a$ \\
\hline 3 Café sem açúcar & $4,42 \pm 0,8^{b c}$ & $3,14 \pm 0,9^{* b c}$ \\
\hline \multicolumn{3}{|l|}{ Salgado } \\
\hline 1 Biscoito de sal & $3,00 \pm 1,2$ & $3,42 \pm 1,4$ \\
\hline 2 Biscoito de polvilho & $2,46 \pm 1,1$ & $3,18 \pm 1,0^{*}$ \\
\hline 3 Azeitona & $4,22 \pm 1,1^{b c}$ & $4,54 \pm 0,9^{b c}$ \\
\hline \multicolumn{3}{|l|}{ Azedo } \\
\hline 1 Suco de limão & $4,42 \pm 0,9$ & $2,86 \pm 1,0^{*}$ \\
\hline 2 Suco de maracujá & $3,76 \pm 1,2^{a}$ & $2,56 \pm 1,0^{*}$ \\
\hline 3 logurte natural & $3,48 \pm 1,2^{\mathrm{b}}$ & $4,49 \pm 0,7^{* \mathrm{bc}}$ \\
\hline
\end{tabular}

Nota: Diferença entre pacientes e controle: ${ }^{*} \mathrm{p}<0,05$. Diferenças dentro do mesmo grupo em relação à intensidade de sabores dos grupo de alimentos doce, amargo, salgado e azedo: 1 vs. $2:{ }^{\mathrm{a}} \mathrm{p}<0,05 ; 1$ vs. $3:{ }^{\mathrm{b}} \mathrm{p}<0,05 ; 2$ vs. $3:{ }^{\mathrm{c}} \mathrm{p}<0,05$.

\section{DISCUSSÃO}

O presente estudo constatou que a maior parte dos pacientes em tratamento oncológico era do sexo feminino e que, nesse grupo, a neoplasia mais frequente foi o câncer de mama. Esses dados corroboram a estimativa publicada pelo Instituto Nacional de Câncer José Alencar Gomes da Silva (INCA), que mostra que o câncer de mama continua sendo o mais incidente em mulheres ${ }^{2}$.

A respeito do público masculino, os achados deste estudo contrariaram a estimativa nacional, que evidencia que o câncer de próstata é o mais incidente entre os homens. No presente estudo, a maior ocorrência de câncer nos homens foi no cólon e reto, a segunda localização mais comum nesse público, com projeção de 41 mil novos casos por $\mathrm{ano}^{2}$. De acordo com a International Agency for Research on Cancer $(\operatorname{Iarc})^{12}$, no ano de 2020, são esperados mais de 1,1 milhão novos casos de câncer de cólon e mais de 740 mil novos casos de câncer de reto. As projeçóes dessa instituição para o ano de 2040 sugerem que esses números irão aumentar cerca de $60 \%$, evidenciando a magnitude desse problema de saúde pública ${ }^{12}$.

A respeito da avaliação antropométrica, o IMC mostrou que a maior parte dos pacientes oncológicos estava acima do peso no presente estudo. Miranda et al. ${ }^{13}$, ao avaliarem 
uma populaçáo semelhante aos pacientes oncológicos da presente pesquisa, também encontraram maior índice de excesso de peso de acordo com a classificação do IMC, em comparação à subnutrição ${ }^{13}$. Esse resultado parece refletir o cenário apresentado pelos demais indivíduos no país e pelo grupo controle. De acordo com os dados publicados em 2020 pelo Vigitel ${ }^{14}$, um inquérito telefônico realizado pelo Ministério da Saúde, a frequência de excesso de peso no Brasil foi de 55,4\% no ano de 2019.

Mas, apesar do excesso de peso constatado, pôde-se notar que os parâmetros que avaliam a massa magra corporal, como a $\mathrm{CB}$ e a $\mathrm{CP}$, estiveram inadequados em alguns pacientes do presente estudo, especialmente ao serem comparados com o grupo controle. Vale mencionar que a inadequação nutricional foi ainda maior nos indivíduos mais velhos, em ambos os grupos, e isso pode ser associado à depleção muscular própria da senilidade. Pesquisadores afirmam que, antes mesmo que a depleção ocorra, a perda da força muscular decorrente do envelhecimento, denominada dinapenia, já está instalada e se associa a desfechos negativos como declínio funcional e óbito ${ }^{15}$.

Estudiosos que realizaram um estudo multicêntrico com indivíduos em tratamento oncológico em mais de 42 hospitais do Brasil e os estratificaram por faixa etária encontraram que aqueles mais velhos apresentaram maior risco para subnutrição. $\mathrm{O}$ estudo ainda evidenciou que os sintomas gastrointestinais decorrentes dos efeitos colaterais do tratamento também foram mais incidentes nos indivíduos com mais de 50 anos, especialmente a falta de apetite ${ }^{16}$.

Sabe-se que os efeitos colaterais dos tratamentos antineoplásicos colaboram para a deterioração do estado nutricional, além do catabolismo produzido pela própria doença. No que diz respeito à disgeusia, esta pode prejudicar a aceitação alimentar dos pacientes em tratamento oncológico ${ }^{10}$.

No presente estudo, os pacientes em tratamento oncológico perceberam o sabor dos seguintes alimentos de maneira mais acentuada: doce de leite, goiabada, suco de limão, suco de maracujá e café.

Os alimentos doces foram percebidos com maior intensidade, além de terem sido avaliados de forma mais uniforme entre os pacientes oncológicos, quando comparados com o grupo controle, no qual a intensidade do sabor doce das amostras variou mais. A percepção mais intensa do sabor doce pelos pacientes em quimioterapia já havia sido reportada por outros estudiosos. Em uma investigação usando soluçôes de cinco intensidades diferentes dos sabores básicos: doce, amargo e umami, os autores constataram que o sabor doce foi percebido de maneira significativamente superior. Eles ainda mostraram que essa diferença impactou negativamente no consumo energético e nutricional dos pacientes ${ }^{17}$.

Os alimentos biscoito de polvilho, iogurte natural, rúcula e chicória foram percebidos com menor intensidade pelos pacientes do presente estudo, quando comparados ao grupo controle.

Dois dos alimentos salgados, o biscoito salgado e o biscoito de polvilho, foram os únicos que não se classificaram na maior intensidade de sabor pela maioria dos pacientes oncológicos. Tal resultado se repetiu também no grupo controle e pode estar associado ao paladar adaptado ao elevado consumo de sódio. Dados da Pesquisa Nacional de Saúde de $2013^{18}$ mostraram que a média de consumo de sódio pela população brasileira foi superior a 9 gramas por dia, ultrapassando o limite superior estipulado pela Organização Mundial da Saúde ${ }^{19}$. Além disso, trata-se de produtos processados, os quais apresentam um consumo frequente e crescente entre os brasileiros ${ }^{20}$.

Vale mencionar que, em um estudo que avaliou a aceitaçấo de preparaçóes por pacientes em tratamento de câncer, nenhum deles relatou sentir o sabor salgado ao degustar uma das receitas ${ }^{7}$, reforçando que talvez o paladar desses indivíduos já esteja mesmo acostumado com um sabor mais acentuado do sal, como o dos demais brasileiros.

Entre os sabores azedo e amargo, dois alimentos se destacaram por apresentarem resultados totalmente opostos entre os grupos de pacientes e controle. Enquanto o grupo controle classificou o iogurte natural como alimento mais azedo e o café sem açúcar como o alimento menos amargo, os pacientes oncológicos disseram o contrário.

A percepção mais amarga do café referida pelos pacientes concorda com os achados de uma pesquisa com pacientes oncológicos, que objetivou avaliar a perda de sensibilidade do paladar para o gosto amargo utilizando também o café. Os autores encontraram a maior sensibilidade para esse sabor, quando comparado ao doce e ao umami ${ }^{21}$.

Este estudo é pioneiro em avaliar a percepção dos sabores por meio de amostras de alimentos. Tal característica é positiva por trazer novos elementos para o estudo da disgeusia em pacientes oncológicos; no entanto, apresenta limitaçóes por não ter investigado as demais variáveis que poderiam influenciar na percepção do paladar desses indivíduos concomitantemente, bem como a interferência da localizaçáo da neoplasia e do tempo de tratamento. Acrescenta-se ainda que a falta de ferramentas padronizadas para a avaliação da percepção do paladar foi outra limitação importante na presente pesquisa.

Os autores que avaliaram as alteraçôes nos sentidos de paladar e olfato, antes e durante o tratamento de 
pacientes com câncer de pulmão, observaram que não apenas a intensidade, mas também as características dessas alteraçóes, podem mudar ao longo do tratamento. Os pesquisadores ainda afirmaram que os pacientes experimentam estas de forma individual e o impacto delas dependeu também dos demais sintomas, efeitos colaterais sofridos e situaçóes de vida em geral ${ }^{22}$.

\section{CONCLUSÃO}

O presente estudo identificou diferenças significativas na intensidade da percepção do paladar entre indivíduos sadios e em tratamento oncológico. Os sabores percebidos com maior intensidade foram os dos alimentos doces, enquanto os dos alimentos do grupo salgado foram percebidos com menor intensidade. Os alimentos azedo e amargo foram notados com intensidades contrárias entre pacientes e indivíduos do grupo controle e não houve associação entre as variáveis clínicas e antropométricas com a percepção do paladar.

Estudos que avaliem as alteraçóes de paladar nos pacientes em tratamento oncológico devem ser estimulados para que se possam minimizar os efeitos adversos da disgeusia e promover maior prazer e satisfaçáo nos momentos de alimentação, colaborando para a preservaçáo do estado nutricional.

\section{CONTRIBUIÇÕES}

Todos os autores contribuíram na concepção e/ ou no planejamento do estudo; na obtenção, análise e interpretação dos dados; assim como na redação e revisão crítica; e aprovaram a versão final a ser publicada.

\section{AGRADECIMENTOS}

Às alunas do Curso de Nutrição da Universidade de Franca, Gabriela Freitas Cintra e Gabriela Samara Gonçalves Xavier, pela participação na coleta dos dados do grupo controle.

\section{DECLARAÇÃO DE CONFLITO DE INTERESSES}

Nada a declarar.

\section{FONTES DE FINANCIAMENTO}

Coordenação de Aperfeiçoamento de Pessoal de Nível Superior (Capes) - Código de Financiamento 001.

\section{REFERÊNCIAS}

1. Goss PE, Lee BL, Badovinac-Crnjevic T, et al. Planejamento do controle do câncer na América Latina e no Caribe. Lancet Oncol [Internet]. 2013[acesso 2020 jan 20];14:391-436. Disponível em: https://els-jbs-prodcdn.jbs.elsevierhealth.com/pb/assets/raw/Lancet/stories/ commissions/planning-cancer-control-latin-americaand-caribbean/tlo-commission-series-portuguese.pdf

2. Instituto Nacional de Câncer José Alencar Gomes da Silva. Estimativa 2020: incidência de câncer no Brasil [Internet]. Rio de Janeiro: INCA; 2019 [acesso 2020 jan 20]. Disponível em: https://www.inca.gov.br/sites/ ufu.sti.inca.local/files/media/document/estimativa-2020incidencia-de-cancer-no-brasil.pdf

3. Instituto Nacional de Câncer José Alencar Gomes da Silva [Internet]. Rio de Janeiro: INCA; [data desconhecida]. Câncer: o que é câncer?; 2020 [acesso 2020 jan 20]. Disponível em: https://www.inca.gov.br/o-que-e-cancer

4. Prado BBF. Influência dos hábitos de vida no desenvolvimento do câncer. Cienc Cult. 2014;66(1):2124. doi: http://doi.org/10.21800/S000967252014000100011

5. Poltronieri TS, Tusseti C. Impacto do tratamento do câncer sobre o estado nutricional de pacientes oncológicos: atualizaçáo da literatura. Rev Bras Ciên Saúde 2016;20(4):327-32.

6. Silva AIV, Galante C, Manzi FR. Efeito da radiação ionizante sobre o paladar em pacientes submetidos a radioterapia para a regiáo da cabeça e pescoço. Radiol Bras. 2011;44(5):297-300. doi: https://doi.org/10.1590/ S0100-39842011000500007

7. Palmieri BN, Moulatlet EM, Buschinelli LKO, et al. Aceitação de preparaçóes e sua associação com os sintomas decorrentes do tratamento de câncer em pacientes de uma clínica especializada. Cad Saúde Colet. 2013;21(1):2-9. doi: https://doi.org/10.1590/S1414462X2013000100002

8. Alexandre TS, Duarte YAO, Santos JLF, et al. Prevalência e fatores associados à sarcopenia, dinapenia e sarcodinapenia em idosos residentes no Município de São Paulo - Estudo SABE. Rev Bras Epidemiol. 2018;21(Supl.2):1-13. doi: https://doi.org/10.1590/1980-549720180009.supl.2

9. Barragán R, Coltell O, Portolés O, et al. Bitter, sweet, salty, sour and umami taste perception decreases with age: sex-specific analysis, modulation by genetic variants and taste-preference associations in 18 to 80 year-old subjects. Nutrients. 2018;10(10):1539. doi: https://doi. org/10.3390/nu10101539

10. Murtaza B, Hichami A, Khan AS, et al. Alteration in taste perception in cancer: causes and strategies of treatment. Front Physiol. 2017;8:134. doi: https://doi.org/10.3389/ fphys.2017.00134

11. Pugnaloni S, Vignini A, Borroni F, et al. Modifications of taste sensitivity in cancer patients: a method for the evaluations of dysgeusia. Support Care Cancer. 2020;28(3):1173-81. doi: https://doi.org/10.1007/ s00520-019-04930-x 
12. Ferlay J, Laversanne M, Ervik M, et al. Global Cancer Observatory: Cancer Tomorrow [Internet]. Lyon, France: International Agency for Research on Cancer; c1965-2020 [cited 2020 Feb 26]. Available from: http:// gco.iarc.fr/tomorrow/graphic-isotype?type $=0$ \&popul ation $=900 \&$ mode $=$ population $\&$ sex $=0 \&$ cancer $=39 \& a$ ge $\_$group $=$value $\& a p c \_$male $=0 \&$ apc $\_f e m a l e=0$

13. Miranda TV, Neves FMG, Costa GNR, et al. Estado nutricional e qualidade de vida de pacientes em tratamento quimioterápico. Rev Bras Cancerol. 2013;59(1):57-64. doi: https://doi.org/10.32635/21769745.RBC.2013v59n1.544

14. Ministério da Saúde (BR), Secretaria de Vigilância em Saúde, Departamento de Análise em Saúde e Vigilância de Doenças não Transmissíveis. Vigitel Brasil 2019: vigilância de fatores de risco e proteção para doenças crônicas por inquérito telefônico: estimativas sobre frequência e distribuição sociodemográfica de fatores de risco e proteção para doenças crônicas nas capitais dos 26 estados brasileiros e no Distrito Federal em 2019. Brasília, DF: Ministério da Saúde, Secretaria de Vigilância em Saúde, Departamento de Análise em Saúde e Vigilância de Doenças não Transmissíveis; 2020.

15. Manini TM, Clark BC. Dynapenia and aging: an update. J Gerontol A Biol Sci Med Sci. 2012;67(1):28-40. doi: https://doi.org/10.1093/gerona/glr010

16. de Pinho NB, Martucci RB, Rodrigues VD, et al. High prevalence of malnutrition and nutrition impact symptoms in older patients with cancer: results of a Brazilian multicenter study. Cancer. 2020;126(1):15664. doi: https://doi.org/10.1002/cncr.32437

17. Sanchéz-Lara K, Sosa-Sanchéz R, Green-Renner D, et al. Influence of taste disorders on dietary behaviors in cancer patients under chemotherapy. Nutr J. 2010;9:15. doi: https://doi.org/10.1186/1475-2891-9-15

18. Mill JG, Malta DC, Machado IE, et al. Estimativa do consumo de sal pela populaçáo brasileira: resultado da Pesquisa Nacional de Saúde 2013. Rev Bras Epidemiol. 2019;22(Suppl 2):1-14. doi: https://doi. org/10.1590/1980-549720190009.supl.2

19. World Health Organization. Guideline: sodium intake for adults and children [Internet]. Genebra: WHO; 2012 [cited 2020 Jan 20]. Available from: https://www.who. int/publications/i/item/9789241504836

20. Instituto Brasileiro de Geografia e Estatística, Coordenação de Trabalho e Rendimento. Pesquisa de orçamentos familiares 2017-2018: avaliação nutricional da disponibilidade domiciliar de alimentos no Brasil. Rio de Janeiro: IBGE; 2020.

21. Caldas APS. Associação entre perda do paladar para gostos básicos (doce e amargo) e umami e características clínicas e nutricionais de pacientes oncológicos sob quimioterapia [monografia]. São Luís, MA: Universidade Federal do Maranhão; 2014.
22. Belqaid K, Tishelman C, McGreevy J, et al. A longitudinal study of changing characteristics of self-reported taste and smell alterations in patients treated for lung cancer. Eur J Oncol Nurs. 2016;21:232-41. doi: http://doi. org/10.1016/j.ejon.2015.10.009

Recebido em 13/5/2020 Aprovado em 22/9/2020 